UCRL-ID-120286

\title{
Monte Carlo Study of a High-Sensitivity Gamma-Ray Detection System
}

D. J. Decman and M. N. Namboodiri

Nuclear Science Applications Section

Isotope Sciences Division

Chemistry \& Materials Science

Lawrence Livermore National Laboratory

March 1995

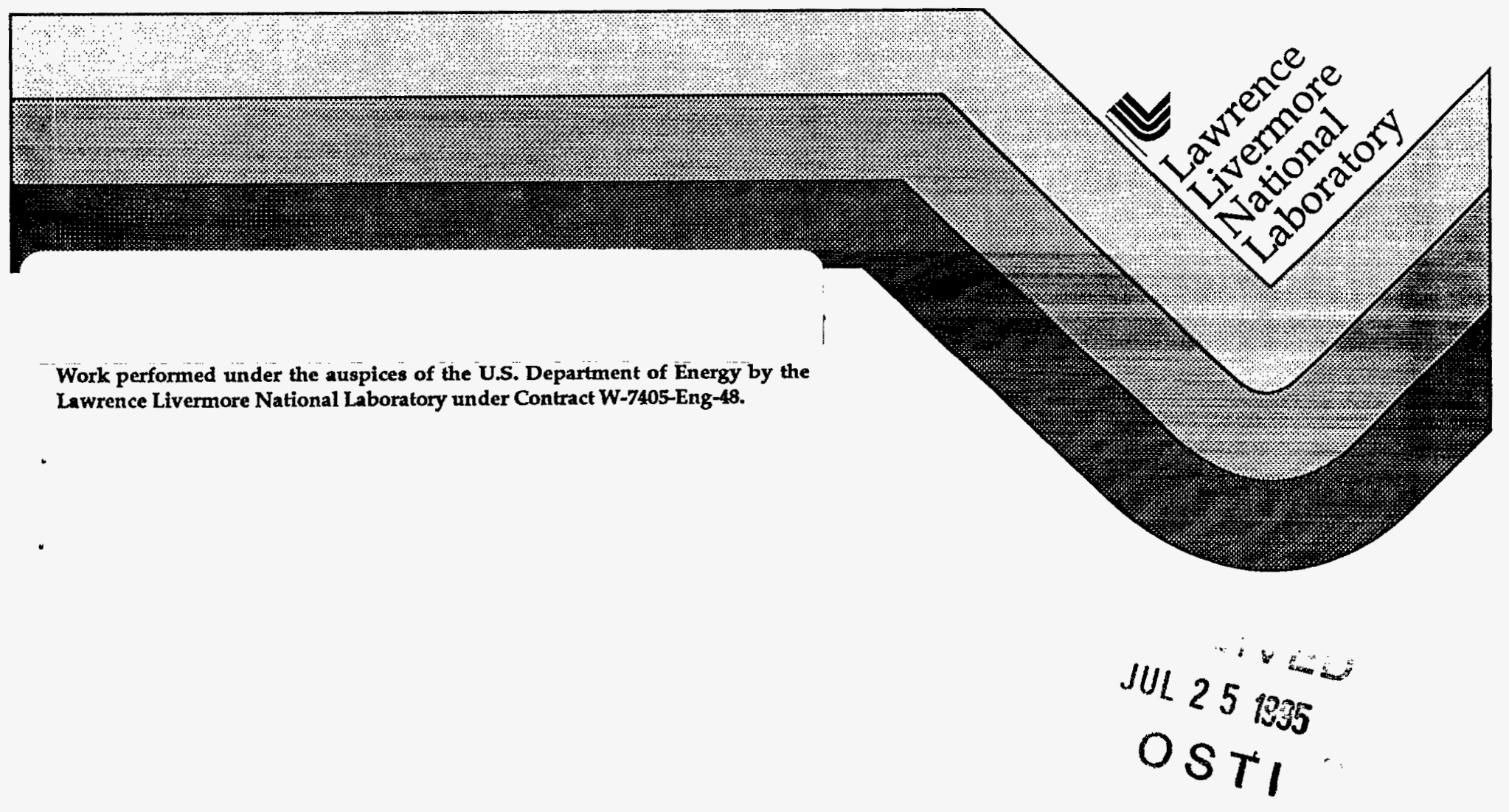




\section{DISCLAIMER}

This document was prepared as an account of work sponsored by an agency of the United States Government. Neither the United States Government nor the University of California nor any of their employees, makes any warranty, express or implied, or assumes any legal liability or responsibility for the accuracy, completeness, or usefulness of any information, apparatus, product, or process disclosed, or represents that its use would not infringe privately owned rights. Reference herein to any specific commercial products, process, or service by trade name, trademark, manufacturer, or otherwise, does not necessarily constitute or imply its endorsement, recommendation, or favoring by the United States Government or the University of California. The views and opinions of authors expressed herein do not necessarily state or reflect those of the United States Government or the University of California, and shall not be used for advertising or product endorsement purposes.

This report has been reproduced directly from the best available copy.

Available to DOE and DOE contractors from the

Office of Scientific and Technical Information

P.O. Box 62, Oak Ridge, TN 37831

Prices available from (615) 576-8401

Available to the public from the

National Technical Information Service

U.S. Department of Commerce

5285 Port Royal Rd.

Springfield, VA 22161 


\section{DISCLAIMER}

Portions of this document may be illegible in electronic image products. Images are produced from the best available original document. 


\title{
Monte Carlo Study of a High-Sensitivity Gamma-Ray Detection System
}

\author{
D. J. Decman and M. N. Namboodiri \\ Isotope Sciences Division \\ Lawrence Livermore National Laboratory \\ Livermore, CA 94550
}

\begin{abstract}
:
We use Monte Carlo calculations to study a new design for a highsensitivity gamma-ray detection system. The system uses an array of highpurity germanium detectors operating with a event-mode data acquisition system. The calculations show that the proposed design could produce a factor of 10 increase in the sensitivity of these measurements compared to currently employed systems.
\end{abstract}




\section{Introduction}

Gamma-ray spectroscopy with high-purity germanium (HPGe) detectors has proven to be a useful technique in measuring radioactivity in low level samples. In this report we discuss an innovative multi-detector system that could extend the current capabilities in gamma-ray counting facilities to allow the measurement of very low activities in time-critical situations, such as the analysis of samples with short-lived isotopes or timely political significance. This capability would be essential to new efforts in nuclear forensics in which samples from suspected nuclear events or activities could be analyzed and interpreted quickly to allow national leaders to make timely decisions with confidence.

The sensitivity of a gamma-ray spectroscopy system is usually defined in terms of the minimum detectable activity (MDA). Cooper gives the following expression for the MDA for a gamma ray of energy $E_{1}$ and a counting time $T$ [ref. 1]:

$$
\operatorname{MDA}\left(E_{1}\right)=\frac{A}{\varepsilon_{E_{1}} T}\left\{\left[W\left(E_{1}\right)\left(B_{N}\left(E_{1}\right)+B_{C}\left(E_{1}\right)\right)+\frac{1}{4} A^{2}\right]^{\frac{1}{2}}+\frac{1}{2} A\right\}
$$

where $\varepsilon_{\mathrm{E} 1}$ is the Ge detector efficiency, $\mathrm{W}$ is the peak width in channels for this energy region, $B_{N}\left(E_{1}\right)$ is the natural background/channel and $B_{C}\left(E_{1}\right)$ is the source-produced Compton background/channel. The parameter $\mathrm{A}$ is the reciprocal fractional error of the measurement, i.e., for a $50 \%$ error $A$ has the value of 2 . Given a counting time of $10^{4}$ minutes typical values of the MDA for a $50 \%$ reciprocal error for the detector systems in most counting facilities are of the order of 10 photons/min. One can improve this value by lowering the background of the detector or by increasing the system efficiency. The equation shows that the sensitivity increases linearly with increasing efficiency whereas one only gains by the square root of a background reduction factor.

Throughout the 70's and 80's gamma-ray spectroscopists greatly increased system sensitivity by reducing the background levels of their measurements. This technology was driven by ultra-low background physics experiments, such as the search for double beta decay in $76 \mathrm{Ge}$. The combination of shielding, judicious selection of detector component materials, and suppression of airborne radioactivity can routinely reduce the ambient background rates by 100 . The systems now used by most counting facilities have applied these measures so that significant gains in background reduction will require more drastic measures.

However huge gains could be made by improvements to the efficiency. The typical efficiency of a standard HPGe counting systems for $500 \mathrm{keV}$ gamma rays is only 0.005 . This low value is due to the relatively small solid angle in most detector systems 
and the small photoelectric absorption coefficient. Most gamma rays above $300 \mathrm{keV}$ interact by Compton scattering so there is a very large probability that only a fraction of the energy will be deposited in the detector. These Compton scatter events then become background for lower energy gamma rays in the spectrum. This is the $B_{C}\left(E_{1}\right)$ term in the equation above.

One approach to achieving high efficiency for HPGe detectors is the use of the well geometry. In this design the sample can be positioned inside the coaxial core of the detector thus allowing a very large solid angle. The difficulty with these detectors is the relatively small amount of detector material at the bottom of the well and the restrictions on the size of the sample due to small diameter of the well. In addition there are severe summing corrections when measuring those isotopes whose decay involves the emission of two or more gamma-rays in coincidence. This summing greatly complicates the interpretation of the gamma-ray spectra since one observes "sum peaks" at the energy of the two gamma rays added together. However a greater concern is that a full energy event from one transition will sum with a Compton event in the other thereby effectively decreasing the efficiency for the detection of an isotope.

Our proposed design for a high sensitivity gamma ray detection system is similar to the well detector concept in that we strive to have all the gamma rays emitted by the source interact with high resolution detector material. However we would achieve this by surrounding the source with many germanium crystals. The crystals would be thick enough so that for most gamma rays all the energy should be deposited in the detector material. Operating this device simply as many single detectors would increase the efficiency by more than an order of magnitude because of the $4 \pi$ solid angle. However we propose to use an event-mode data acquisition system to achieve greater sensitivity. In this data acquisition scheme we would operate like a high energy physics experiment; when one crystal detects an event we would read the signals from all the detectors. The energy summed for all the detectors for any event would be the characteristic parameter for an isotope.

This coincidence technique increases the system sensitivity by the Compton addback factor. That is, by positioning the crystals close together one is able to measure the Compton scattering from one crystal to another. In these events the summed energy adds all the multiple scattering events back into the full energy peak. These events would have been lost from the full energy peak in a singles mode operation. This also reduces the interference from Compton scattering from higher energy gamma rays, since these events are also added together to produce higher energy sums. Even larger gains in sensitivity can be expected for isotopes that decay by a cascade of two or more gamma-rays. In this 
case the summed energy would be the total energy of the cascade; additional information would be obtained by the distribution of the energy in the various crystals.

One possibility for the design of this high sensitivity system consists of two detector arrays each composed of seven large-volume germanium crystals. The design would have six crystals in a coaxial ring around a central detector. All of these crystals would be in the same cryostat to minimize the distance and amount of material between the crystals. The central detector would be recessed with respect to the outer detectors. The corresponding depression in the end cap of the array would serve as the sample holder. A second array of the same design would then fit against the end cap of the first array. The volume of the sample holder would be $400 \mathrm{~cm}^{3}$.

In this report we describe the results of Monte Carlo calculations to determine the performance of the proposed concept for low-level detection. We also use these same calculations to test some basic design considerations for possible hardware realizations of these multi-detector arrays.

\section{Monte Carlo Methods for calculation photon detector efficiency}

The determination of the efficiency of a gamma-ray detector is a complex problem that is usually broken down into two components, the solid angle and the detector response. The solid angle is determined by the geometry of the system. For a point source on the axis of a cylindrical detector the solid angle is given by

$$
\Omega=\int_{0}^{\alpha} 2 \pi \sin \theta \mathrm{d} \theta=2 \pi(1-\cos \theta)
$$

where $\tan (\theta)$ is the radius of the detector divided by the distance to the source. However, far more complicated expressions are needed when the point is moved off-axis, Moens et al. describe these equations in reference 2 . These expressions for the solid angle must be solved numerically, and become even more complicated when the source has an extended geometry.

The calculation of the detector response is even more difficult due to the complex nature of the interactions of photons with matter. These interactions include Compton (incoherent) scattering, Rayleigh (coherent) scattering, the photoelectric effect, x-ray fluorescence and, for high energies, pair production. The energy dependence of these interactions for germanium is shown in figure 1. For very low energy photons the interactions are dominated by the photoelectric effect, therefore for energies below 90 $\mathrm{keV}$ the mean free path is so short that the efficiency is simply proportional to the solid angle. However for higher energies the detector response to photons becomes far more complicated so that the solid angle in itself does not really represent the bulk of the 
problem. The true measure of the efficiency is related to those gamma-ray trajectories that actually pass through some minimum thickness of Ge. Also for energies above 100 $\mathrm{keV}$ the production of a full energy peak in the spectrum will seldom be caused by a single photoelectric effect process, rather the photon will undergo several Compton scattering before it produces a photoelectron that is contained in the detector. Therefore the probability of producing a full energy peak depends on the availability of additional Ge around the first interaction point to increase the probability of additional Compton scatters that can also be captured in the crystal.

Because of this "multiple interaction" mechanism of the photons an accurate calculation can only be done by ray-tracing. This involves examining a range of trajectories from the source to the detector element. One would then have to assign a probability of interaction for each position in the detector along that track. Then for each interaction one must calculate the probability for each of the possible processes. Because this will often be Compton scattering one must then track both the electron and the scattered photon. Thus with each interaction the problem becomes increasingly complicated so that a systematic calculation of all trajectories becomes intractable. However we can approximate this exact ray-tracing scheme by Monte Carlo calculations. In this technique we assume that we can choose a large enough sample of these trajectories to understand the detector response.

Our calculations were performed using the program MCNP3. This code was developed at LANL and was obtained from the RSIC computer code collection at ORNL. The code generates histories of photons of a given energy from a user defined source as it traverses through various regions of interest. The history is stopped when the energy is degraded to some low-level cut-off value or when the particle trajectory is such that it could not contribute to the system being studied. As the photon interacts with materials it generates electrons and scattered photons all of which are tracked until they are deemed uninteresting.

Unfortunately MCNP does not have a provision to calculate the detector response to a cascade of coincident gamma-rays. Therefore the calculations we present here do not include any test of the gain in sensitivity from incorporating this feature. However the calculations can test the Compton add-back contribution to the efficiency of a detector array.

MCNP uses a standard set of surfaces to describe the geometry of the system components. These surfaces are planes, spheres, cylinders and cones. They can be aligned with the axes of the coordinate system or have more complex positions. The actual detector components are described in MCNP in terms of cells. The cells are defined by 
using the surfaces as boundaries. MCNP uses a Boolean algebra system to enable these combinations to describe virtually any shape. These cells are then filled with user specified materials to represent the components of interest.

The gamma-ray spectrum and/or the photopeak efficiency of a detector is calculated by means of the pulse height tally in MCNP. This tally simply sums the total energy deposited for each event in a given cell, in our case this is the cell corresponding to the active germanium detector element. The result of this tally is the probability per event, that a given energy is deposited in the detector. MCNP provides the user a wide range of options for the energy bins such that for the photopeak efficiency calculations one can define a suitable bin for the full gamma-ray energy. In this way MCNP can directly output the probability of producing a peak in the detector system.

One should note that the MCNP calculations do not actually reproduce the observed full energy peak intensities, this is because the quantity that is tallied is the energy deposited in the active germanium. Therefore the calculations do not include the actual efficiency of the charge collection process in the crystal. In addition we must use the manufacturer's specifications for the important detector parameters such as the crystal length, radius, size of the core, and the thickness of the dead layer.

MCNP also calculates the variance associated with the probability given for each energy bin. This error is based on the sampling statistics and can be minimized by simply running more particles. By using more particles one increases the population of events that actually interact with the detector and therefore does a better job of sampling the interactions of the system.

MCNP provides a wide range of options in defining the source of the photons. The user can define the starting position, the energy, and the direction of the emission. The parameters can be defined by scalar quantities or distributions. In addition one can choose how these distributions will be sampled. We have used point sources of fixed energy for the calculations described in this report. However for some cases we have used source direction biasing techniques to reduce the variance of the tally. In particular in the calculations for the response of a single detector for $1332 \mathrm{keV}$ gamma-rays we have biased the source to sample only the most interesting $5 \%$ of the $4 \pi$ solid angle available for emission. In this way we are able to adequately sample the system response by tracking only $10^{6}$ photons per problem. These calculations can be done in a few minutes on SPARCStation II computers. 


\section{Monte Carlo Test Cases}

We have performed a series of MCNP calculations on single detector systems to check the accuracy of the code and to establish a baseline on the performance of the systems currently in use. By comparing the calculations of the multi-detector system to these one-detector results we hope to quantify the gain by using these more sophisticated systems.

The IEEE has specified a standard method for quoting the efficiency of a HPGe detector. The efficiency of the system is given for the count rate of the $1332 \mathrm{keV}$ gammaray of a point source of ${ }^{60} \mathrm{Co}$. The source is located on axis at a distance of $25 \mathrm{~cm}$ from the front face of the detector end cap. The result is quoted as the ratio of the response of a 3 by 3 inch NaI detector for the same source and distance. The response for this NaI detector is assumed to be $1.2 \times 10^{-3}$. Therefore a Ge detector with this efficiency at 1332 $\mathrm{keV}$ is said to be a $100 \%$ detector. At the time that this standard was developed most $\mathrm{Ge}$ detectors had relatively small volumes and efficiencies of $10-20 \%$. However as detector fabrication technology has improved the crystal sizes have increased dramatically so that detectors with efficiencies greater than $100 \%$ can be routinely obtained.

As a first test of MCNP we have calculated the efficiency of a $3 \times 3$ inch NaI detector for the $1332 \mathrm{keV}$ line of ${ }^{60} \mathrm{Co}$, i. e. the IEEE standard definition. For this simple test we describe the NaI detector a uniform cylinder of $\mathrm{NaI}$ with a density of $3.67 \mathrm{~g} / \mathrm{cm}^{3}$. We sample $2.5 \%$ of the full solid angle by directionally biasing the source trajectories toward the crystal in this way we can achieve good sampling statistics with $10^{6}$ particles. The efficiency is then given by the probability in the pulse height tally for the bin at the full energy peak after correcting for the full solid angle. The MCNP result is $1.35 \times 10^{-3}$ with a sampling error of $0.4 \%$. This is remarkably close to the accepted value considering that this calculation assumes a perfect detector response for the energy deposited in the crystal.

In a similar manner we can test the MCNP code by calculating the efficiency of actual HPGe detectors and comparing the results to the measured efficiency at $1332 \mathrm{keV}$. However the HPGE detector structure is more complicated than the NaI crystal so the MCNP description is quite involved. We therefore offer a more detailed account of these structures both for the understanding of the limitations of the calculations and to provide some important facts for actual detector system design.

A coaxial detector is formed from a cylinder of high-purity germanium. The cylinder is cut to length and milled to the specified diameter. The front face of these detectors are beveled or "bulletized". This gives the detector better charge collection properties. An $8 \mathrm{~mm}$ diameter hole is then drilled into the center of the back side of the 
cylinder creating a hollow core that extends to within $1 \mathrm{~cm}$ of the front face. This core will become the inner contact for the electric field. For $\mathrm{p}$-type detectors the $\mathrm{n}^{+}$contact is formed by diffusing lithium over all the outside surfaces except the flat (unbeveled) back face. The lithium diffused contact has a thickness of approximately $700 \mu \mathrm{m}$, thus for $\mathrm{p}$ type detectors an appreciable dead layer of germanium must be penetrated by the gamma radiation. This dead layer is quite significant for gamma-ray energies less than $100 \mathrm{keV}$. The inner contact for p-type detectors is an ion-implanted boron layer with a thickness less than $0.5 \mu \mathrm{m}$. For $n$-type detectors the contact materials are switched, thus allowing good low-energy photon response for these detectors. After the contacts have been produced, the outside detector surfaces are passivated with a coating of amorphous germanium hydride.

The detector element is housed in a thin-walled aluminum mounting cup. This cup will form the electrical contact to the high voltage and the thermal contact to the cooling rod. The mounting cup is attached to the copper cooling rod through an aluminum oxide disk which affords good thermal conduction and electrical isolation. This assembly is covered with an end cap of a low- $Z$ material, usually aluminum, to create a chamber for high vacuum.

For Monte Carlo simulations we can do a reasonably good job approximating the detector element as a combination of concentric cylindrical surfaces with three different structures along the length of the crystal. The first section is the dead layer at the front of the detector, this is simply a cylinder with the manufacturer's stated crystal diameter and the length of the dead layer thickness. The next section is that part of the detector element that does not have a core, i. e. the front centimeter of the detector. This is composed of two cylinders, one with the crystal diameter to represent the outside of the dead layer and a second cylinder with a diameter equal to the crystal diameter less twice the stated dead layer thickness, this represents the active germanium crystal in this section. The final section of the modeled detector has the same two cylinders as the previous part but also has third cylinder with a diameter of $8 \mathrm{~mm}$ to represent the empty core. In a similar way we can represent the aluminum mounting cup and end cap as a collection of somewhat larger cylinders. We use the manufacturer's specifications for the relative spacing between these components.

We tested the MCNP code by calculating the efficiency of actual HPGe detectors and comparing the results to the measure efficiency at $1332 \mathrm{keV}$. Table 1 shows the manufacturer's specifications of several detectors along with the MCNP calculated efficiency. Also figure 2 shows a plot of the calculated efficiencies as a function of the measured value. Also shown is the result of a straight line fit to these data. The relationship is surprisingly linear with the calculated values $\sim 17 \%$ greater than the 
measured value. Also shown is the result of a straight line fit to these data. The relationship is surprisingly linear with the calculated values $\sim 17 \%$ greater than the measurements. One would expect than the calculated values would be high because of the assumptions involved in equating the pulse height tally with the production of the photopeak. In addition the MCNP description of the actual crystal is only approximate, in particular there is no provision to "bevel" the front edge of the detector. However, since these data span a wide range of detector volumes the linear nature of the offset seems to indicate that a bulk phenomenon is involved as opposed to "edge" or coaxial core effects.

\section{Multi-detector calculations}

Several different arrays of Ge detector elements were studied as possible configurations for the high sensitivity detector system. Monte Carlo calculations of the efficiency of these configurations were performed using the MCNP code. In particular, arrays of hexagonal prisms and cylinders were considered. We also explored a less expensive design that had only 8 detector elements. Finally we performed calculations involving some details of the final construction of the proposed detector array. These issues included the choice of p- or n-type germanium crystals and the possibility of using separate detector capsules in the array.

Detector elements with hexagonal cross section can be packed compactly with relatively little dead space providing high overall efficiency. The array shown schematically in Fig $3 \mathrm{~A}$ has two hexagonal elements facing each other with a gap between them for positioning a source. There is a ring of six hexagonal prisms surrounding each of these two central elements, making an array of 14 elements in all. The calculated efficiency to capture the full energy of gamma rays in such a system as a function of the incident gamma energy is shown in the Fig 3 (diamonds). The particular array used in this calculation had the following dimensions:

Each side of the hexagon:

Length of each element:

$\begin{array}{rr}4 & \mathrm{~cm} \\ 10 & \mathrm{~cm} \\ 5 & \mathrm{~cm}\end{array}$

Gap between the central elements:

$5 \quad \mathrm{~cm}$

(Source gap)

Gap between the outer rings:

$0.5 \mathrm{~cm}$

Gap between adjacent faces of the central and

surrounding elements:

$0.32 \mathrm{~cm}$ 
For simplicity, these elements were assumed to be solid, without the usual coaxial core for making contacts. Also no outer dead layers or end caps were included. Although such calculations overestimate the efficiencies, typically by several per cent, they are useful in evaluating relative efficiencies of different geometrical shapes and arrangements.

For the calculations shown in Fig. 4 and the succeeding figures, a point source at the geometrical center of the array was assumed. However one should note that the efficiency of the detector array improves slightly when the source has an extended geometry. On the other hand the efficiency decreases sharply for a single detector system as one extends the source away from the detector center axis. Therefore the improvement in efficiency is even greater for the extended geometries that would be encountered in counting real samples.

Also shown in Fig. 4 (squares) are efficiencies calculated for an array of two central face-to-face elements surrounded by six elements in the configuration shown in Fig 3B. All eight elements are hexagonal prisms. The dimensions of the elements are the same as before, but the source gap is only $2.5 \mathrm{~cm}$. The two configurations have comparable efficiencies.

The efficiency plotted in Fig. 4 is the fraction of all gamma rays of a given energy emitted by a point source at the center that appears in the full energy peak in a spectrum obtained by coincident summing of all the detector elements. To emphasize the importance of this summing, we note that in the case of the eight element-array, the fractional efficiency of the full energy peak for a $661-\mathrm{keV}$ gamma ray, is 0.089 for each of the two central elements and 0.035 for each of the six outer elements, thus making a total efficiency of only 0.38 if there is no coincident summing; with coincidence one gets the value of 0.63 plotted in Fig 4 .

Fig 4. also illustrates the much higher efficiency of such multi-element arrays compared to that of a single well detector of typical dimensions. For a point source of ${ }^{137} \mathrm{Cs}$, with a gamma-ray energy of $661 \mathrm{keV}$, we find that the well detector is six times less efficient than the array. Again, the results would be even more dramatic for extended sources.

We have also calculated efficiencies for arrays of cylindrical elements. Although they cannot be packed as efficiently as the elements with hexagonal cross section, they are considerably easier to fabricate, especially since the commercial germanium detectors technology uses cylindrical shapes. Fig. 5 shows the geometry of 14-element array in which two central face-to-face cylinders are surrounded by two concentric rings of six cylinders each. The efficiency of such an array is plotted in Fig. 6 for cylinders that are 
$7.5 \mathrm{~cm}$ in diameter and $10 \mathrm{~cm}$ in length. The axes of adjacent cylinders are separated by $7.8 \mathrm{~cm}$ and the source gap (the distance between the faces of the two central elements) is $2.5 \mathrm{~cm}$. The efficiencies obtained by an array with just one ring of six cylinders surrounding the two central elements are also shown. By using two rings of cylinders to minimize gaps in the coverage, one obtains efficiencies comparable to those of the hexagonal arrays. The separation of $7.8 \mathrm{~cm}$ between the axes of adjacent cylinders would be too small if the detector elements are to be enclosed in separate cans (as done in the standard POPTOP detectors from ORTEC, for example). The efficiencies calculated for the same 14-cylinder array with cylinders separated to $9 \mathrm{~cm}$ from each other are also shown. These lower efficiencies would be further suppressed by the addition of material between the cylinders in the form of cans.

Since these cylindrical arrays appear to offer reasonable efficiencies, we made additional calculations using them to obtain more realistic estimates of efficiencies for pand n-type elements. Arrays of 14 cylinders were considered as before. Each element was $10 \mathrm{~cm}$ long, $7.5 \mathrm{~cm}$ in diameter and was enclosed in a mounting cup of $0.5 \mathrm{~mm} \mathrm{Al}$. The results are given in Fig. 7. In the calculation for p-type detectors, the outermost $0.6 \mathrm{~mm}$ of Ge was considered insensitive (this being the usual thickness of the Li-drifted contact region in such detectors). As previously described, this outer contact layer is of negligible thickness in the n-type detectors. For both types, calculations were done for detector elements with and without individual cans. Curves marked A, B and C give comparisons of different cases all with cylinder axes separation at $9.2 \mathrm{~cm}$. The higher efficiency of an n-type array, especially if individual cans are eliminated (using instead a single enclosure for the entire array) is obvious from this figure. In such a system the elements can, of course, be moved closer together to gain additional efficiency as shown by Curve $\mathrm{D}$ for an axis separation of $7.8 \mathrm{~cm}$.

Finally in Fig 7, we also show the efficiency for a system with just two cylindrical detectors corresponding to the two central elements of the p-type array.

\section{Conclusion}

We have performed Monte Carlo calculations to determine the gain in efficiency for multi-detector arrays with coincident summing. The Monte Carlo code was first verified by comparison to single detector measurements. The array calculations show that impressive gains in efficiency can be achieved for both 8 and 14 element detector arrays of hexagonal and cylindrical crystals. We also found that n-type detectors give substantially better efficiency than p-type. 
Several additional aspects of this design must still be studied to understand the effectiveness of these systems. Monte Carlo calculations of coincident gamma rays would be a very important to determine how one could incorporate any additional information from this situation. Such calculations should determine how the distribution of energies in the various detector elements can enhance the sensitivity of the system for certain isotopes. The results reported here on "singles" efficiency" suggest that the coincidence rates should be high enough to make the cascade measurements feasible. Even without this feature we have shown that these multi-element designs with coincident summing can produce at least an order of magnitude improvement in the sensitivity of gamma-ray measurement systems.

\section{References:}

1. J. A. Cooper, Nuclear Instruments and Methods 82, 273 (1970)

2. L. Moens et al., Nuclear Instruments and Methods 187 (1981) 451

3. J. F. Briesmeister et al., Los Alamos National Laboratory Report, LA-7396-M-Rev. 2 (1986)

\section{Figure Captions}

1. Photon cross sections for the photoelectric and Compton effect for germanium.

2. Comparison of measured and MCNP-calculated efficiency for p-type germanium detectors. The solid line is a linear least squares fit to the data, the equation for this line is also shown.

3. Axial and side views of the geometry of the hexagonal prism design for a) 14 and b) 8 detector crystals.

4. Calculated efficiencies for the hexagonal designs and a single well detector.

5. Axial and side view of the geometry of the design with 14 cylindrical detector elements, i. e. two rings of six detectors around the two central detectors.

6. Calculated efficiencies for the cylindrical designs.

7. Calculated efficiencies for cylindrical designs for $n$ - and p-type detectors with an aluminum can, n-type detector calculations with and without cans and at different spacing, also, for comparison, the result for two central detectors only. 
Table 1: Calculated and measured efficiencies for p-type coaxial detecotrs

\begin{tabular}{cccc}
$\begin{array}{c}\text { Diameter } \\
\text { (mm) }\end{array}$ & $\begin{array}{c}\text { Length } \\
\text { (mm) }\end{array}$ & $\begin{array}{c}\text { Measured } \\
\text { Efficiency }\end{array}$ & $\begin{array}{c}\text { MCNP } \\
\text { Efficiency }\end{array}$ \\
\hline 49.9 & 39.5 & 14.7 & 18.0 \\
50.7 & 39.2 & 16.4 & 18.7 \\
54.9 & 45.2 & 21.4 & 27.5 \\
55.9 & 48.5 & 24.3 & 22.5 \\
51.3 & 65.0 & 24.8 & 29.1 \\
72.2 & 59.8 & 58.5 & 74.6 \\
69.9 & 72.4 & 66.7 & 76.7 \\
69.2 & 83.6 & 72.4 & 80.9 \\
71.1 & 94.6 & 75.6 & 91.7 \\
70.0 & 92.6 & 76.9 & 86.9 \\
75.6 & 65.1 & 77.0 & 89.6 \\
81.8 & 55.6 & 79.7 & 96.7 \\
73.8 & 82.3 & 80.7 & 95.6 \\
72.2 & 95.6 & 83.3 & 96.2 \\
73.1 & 90.1 & 83.7 & 96.8 \\
79.0 & 64.3 & 83.7 & 99.2 \\
75.2 & 93.4 & 92.4 & 107.3 \\
75.2 & 100.0 & 95.1 & 110.0 \\
77.0 & 92.5 & 98.3 & 113.9 \\
76.1 & 108.2 & 100.1 & 116.0 \\
79.8 & 94.9 & 110.0 & 126.1 \\
81.7 & 108.6 & 115.3 & 140.0
\end{tabular}




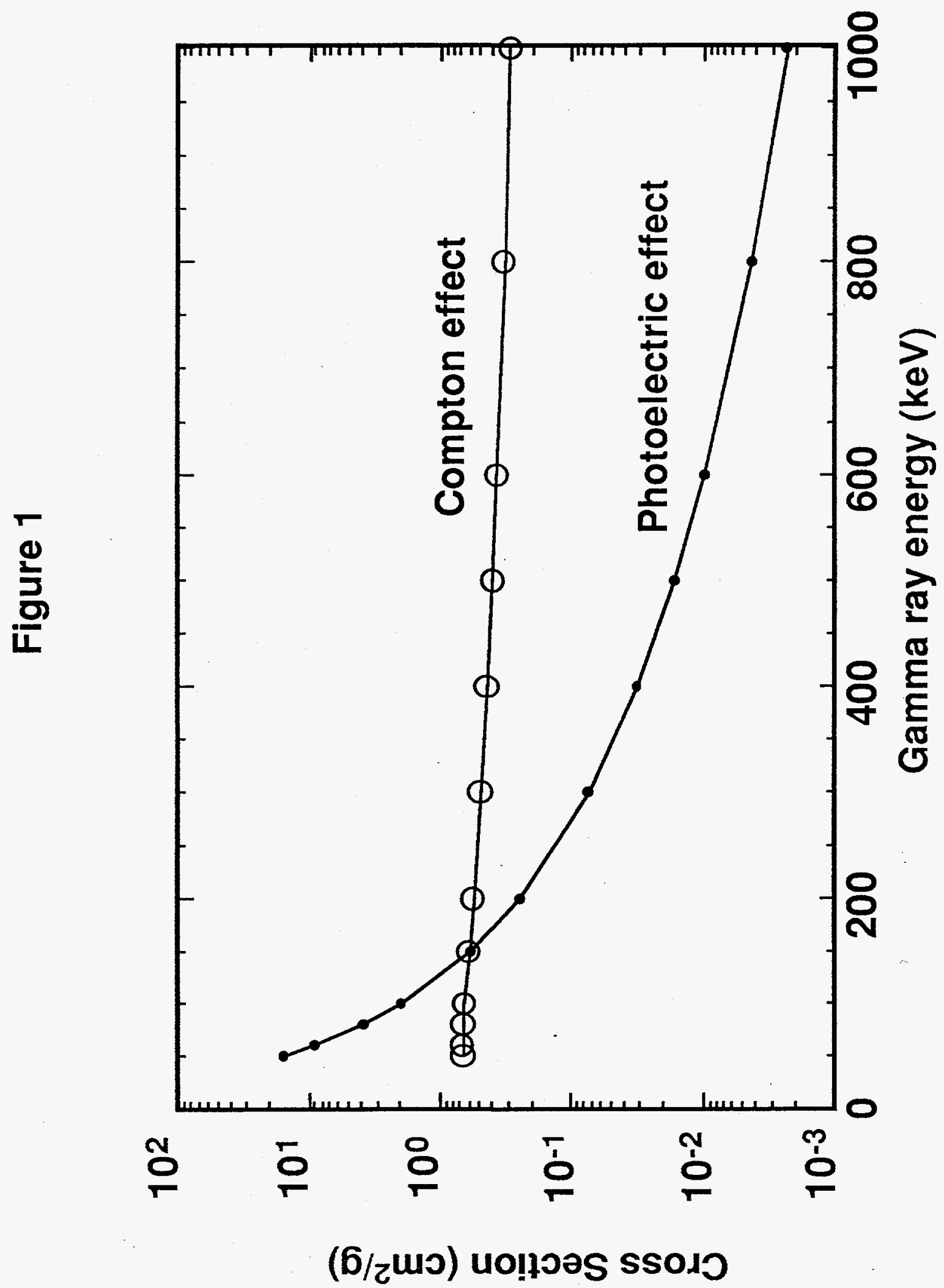




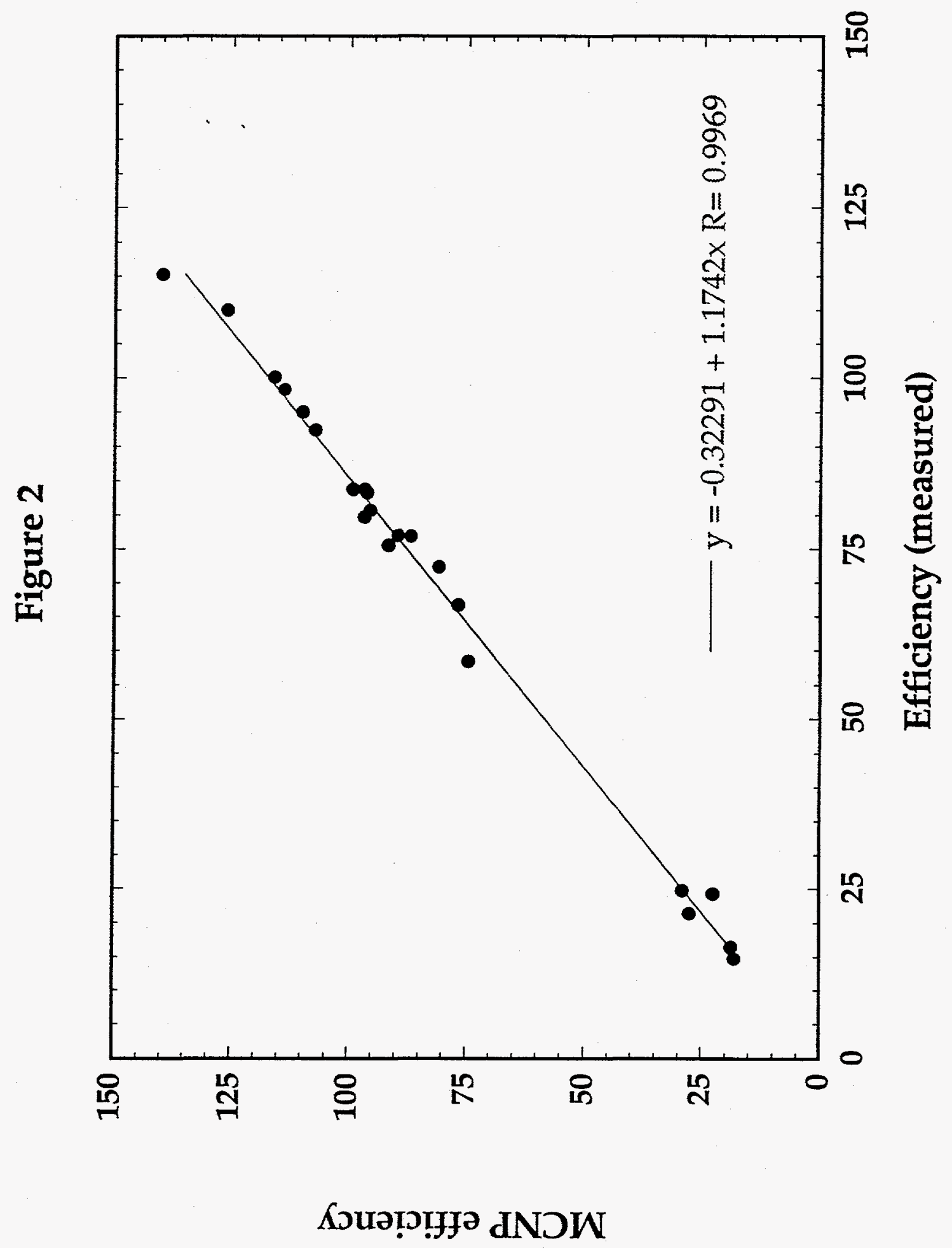



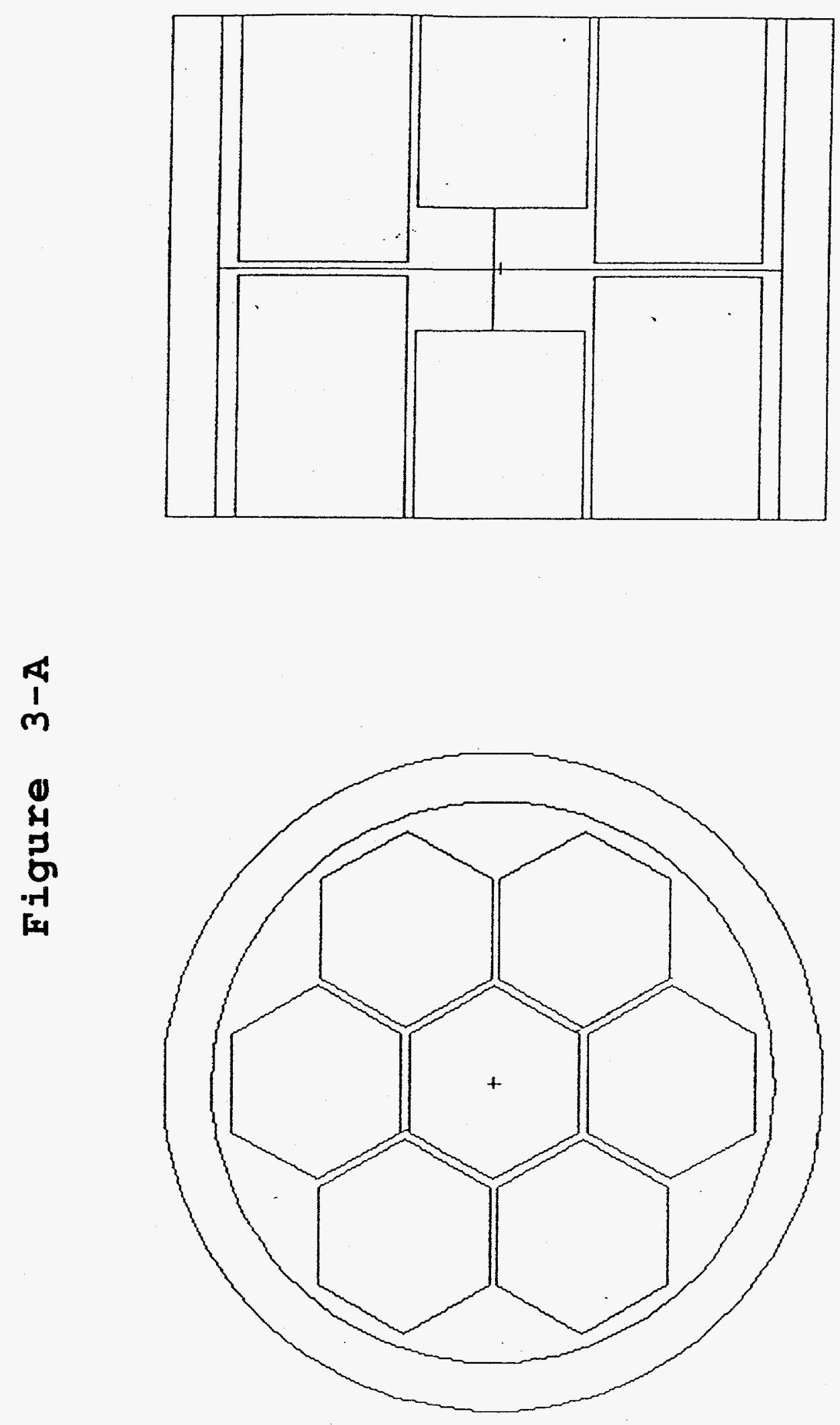


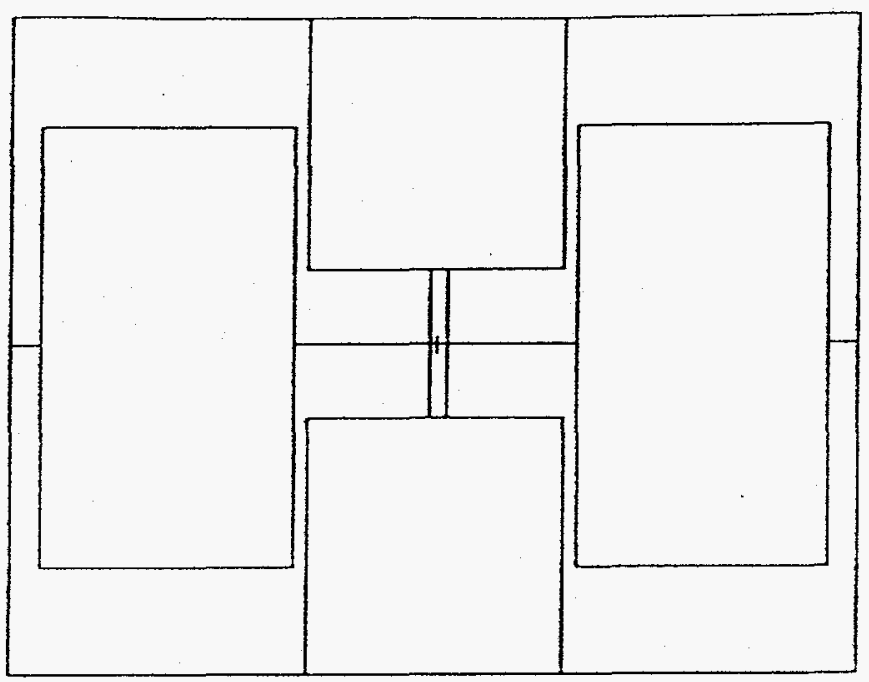

$m$
1
$m$
0
4
0
6
-4
4

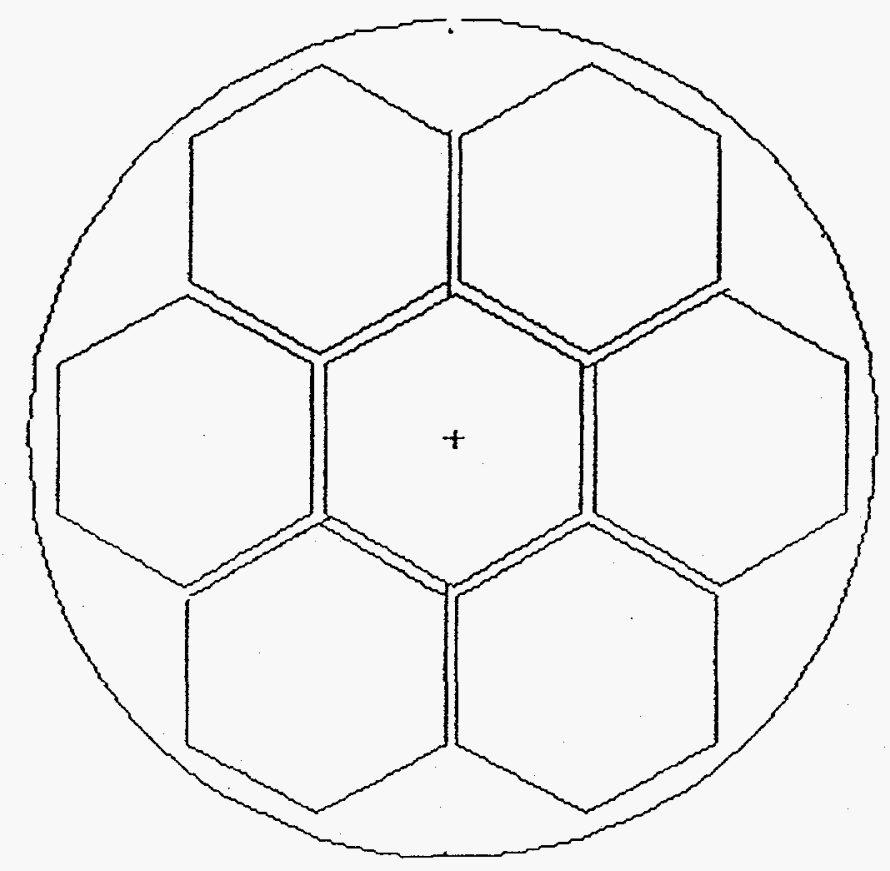




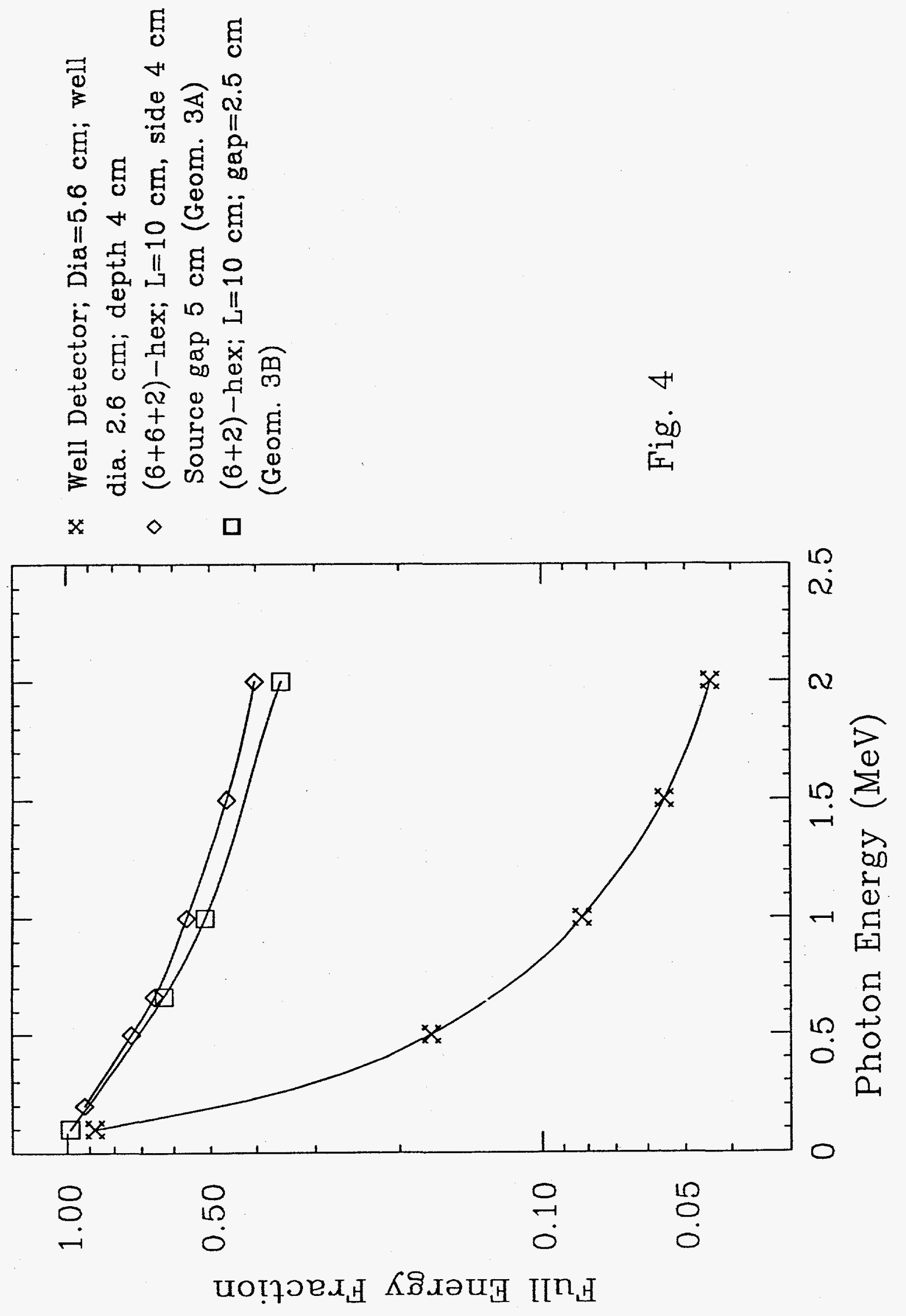



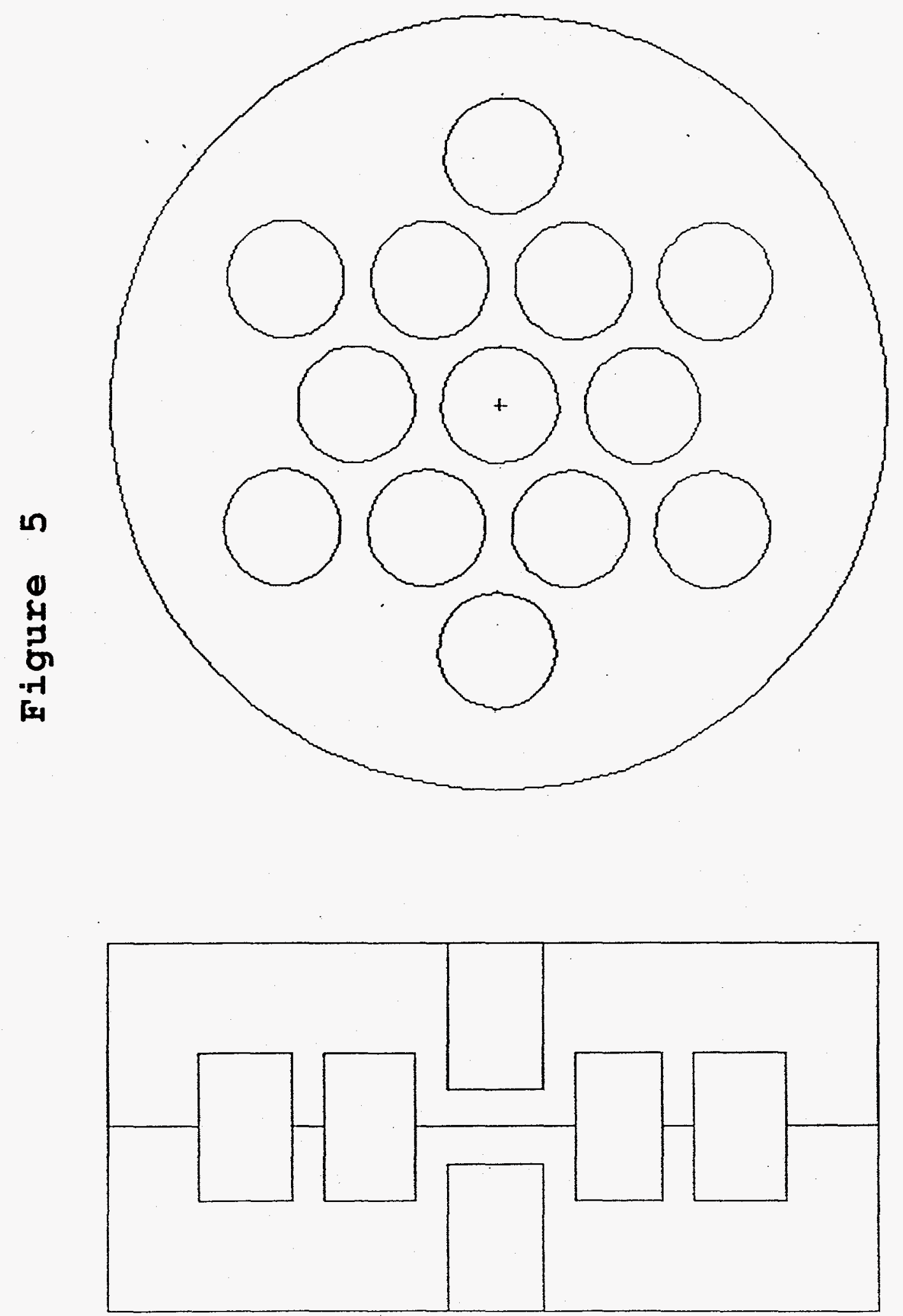

- 19 - 


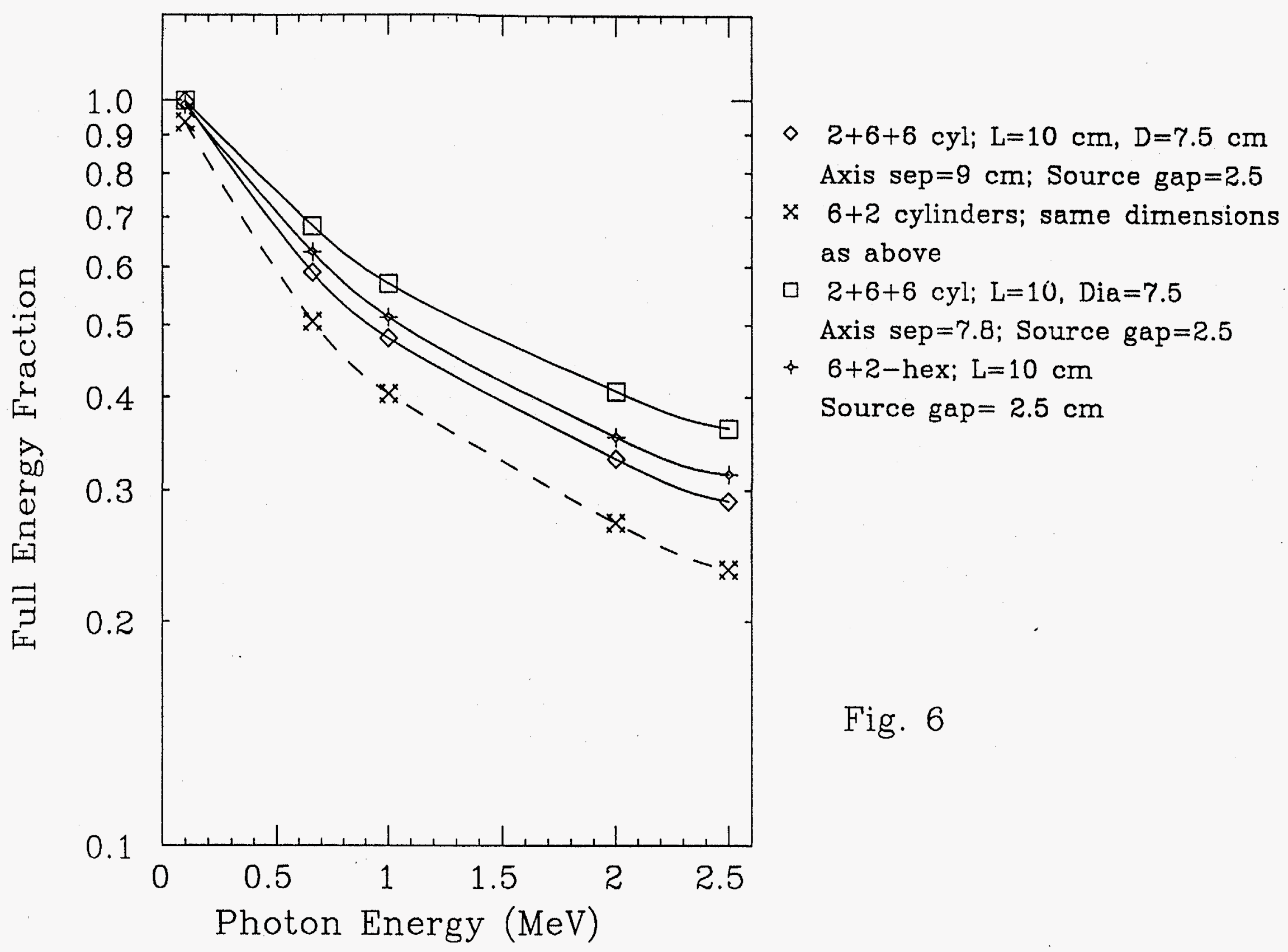




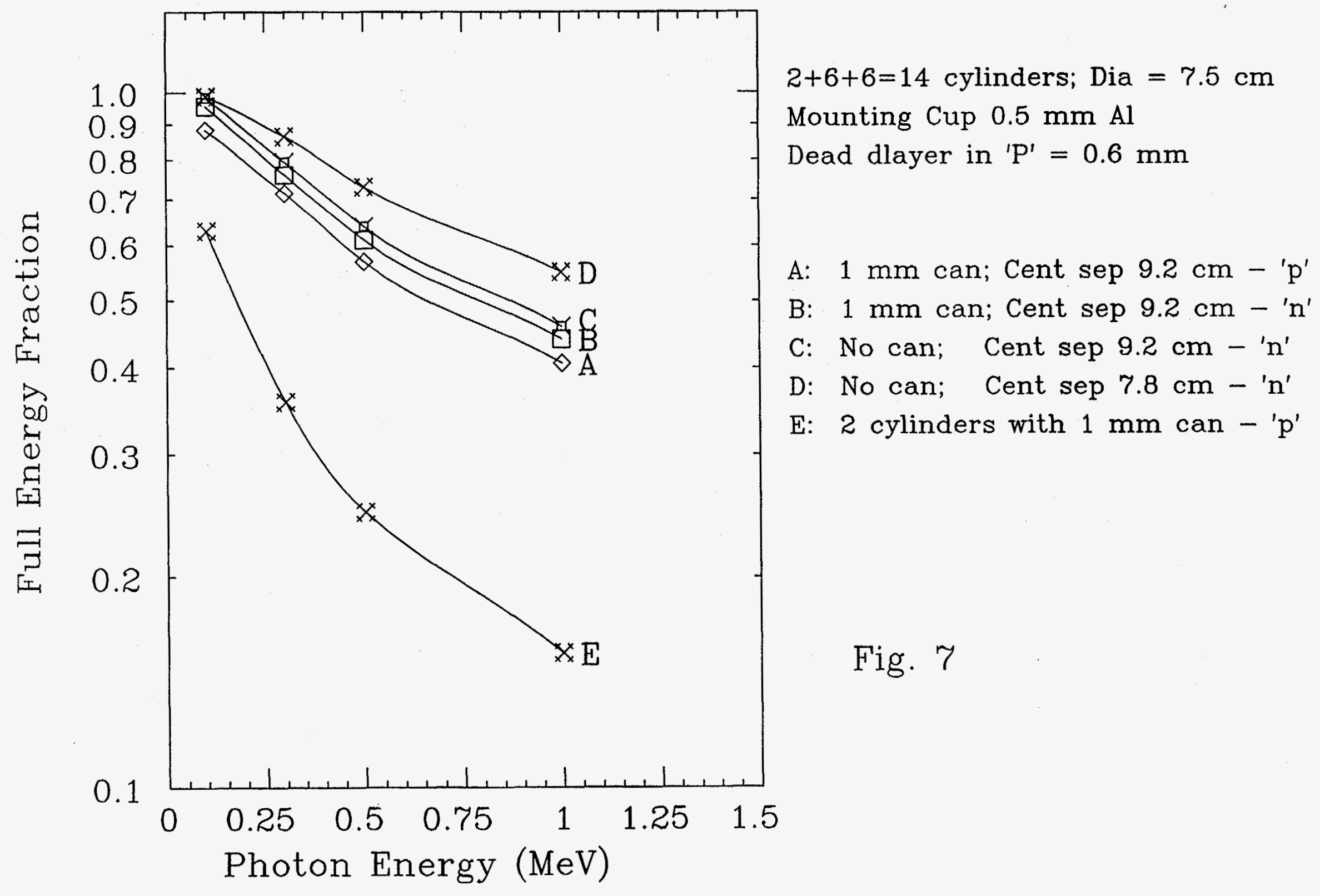

